\title{
Heating of Stellar Chromospheres and Transition Regions
}

\author{
Zdzislaw E. Musielak \\ Department of Physics, University of Texas at Arlington, \\ Arlington, TX 76019, USA \\ email: zmusielak@uta.edu
}

\begin{abstract}
.
To explain the heating of stellar chromospheres and transition regions, two classes of heating mechanisms have been considered: dissipation of acoustic and magnetic waves generated in stellar convection zones; and dissipation of currents generated by photospheric motions of surface magnetic fields. The focus of this paper is on the wave heating mechanisms and on recent results which demonstrate that theoretical models of stellar chromospheres based on the wave heating can explain the "basal flux" and the observed Ca II emission in most stars but cannot account for the observed $\mathrm{Mg}$ II emission in active stars. The obtained results clearly show that the base of stellar chromospheres is heated by acoustic waves, the heating of the middle and upper chromospheric layers is dominated by magnetic waves associated with magnetic flux tubes, and that other non-wave heating mechanisms are required to explain the structure of the highest layers of stellar chromospheres and transition regions.
\end{abstract}

\section{Introduction}

It is now well-established that all late-type stars show chromospheric activity, which is typically identified with the presence of $\mathrm{Ca}$ II $\mathrm{H}+\mathrm{K}$ and $\mathrm{Mg} \mathrm{II} \mathrm{h+k}$ emission lines. Observations also show many UV lines that originate in stellar transition regions located between stellar chromospheres and coronae. The observed activity varies significantly for a given spectral type and decreases when stars move away from the main-sequence (Linsky 1991; Rutten et al. 1991; Stępień 1994; Baliunas et al. 1995; Dupree et al. 1999). For stars that rotate very slow, a low background chromospheric activity (the so-called "basal flux") has been observed (Schrijver 1987). The fact that stars of the same spectral type have different levels of stellar activity is attributed to the presence of surface magnetic fields. Observations show that stars are more active when a larger portion of their surface is covered by the magnetic field and that the effect is especially prominent in fast rotating stars (Linsky 1991).

Direct measurements of stellar magnetic fields demonstrate that the photospheric magnetic flux increases when stellar rotation period is increased (e.g., Saar 1996; Jordan 1997). According to Saar (2001) and Donati (1999), there is growing evidence for inhomogeneous and locally strong magnetic fields in atmospheres of chromospherically active stars but detailed distributions are not 
currently known. For solar-type stars (e.g., Linsky et al. 1992), it is very likely that their magnetic structures resemble those known from the Sun: sunspots and magnetic flux tubes located primarily at the boundaries of supergranules (e.g., Solanki 1993). As a result, stellar atmospheres are likely to have magnetic and non-magnetic regions in their lower parts, and the upper layers being magnetically dominated.

Based on the above observational results, two basic and currently unsolved theoretical problems can be identified. The first one is to predict theoretically the distribution of magnetic fields on the surface of a star with known rotation rate. This requires a first-principle theory of stellar dynamos, which is not yet available (e.g., Weiss 1994). The second is to identify the basic physical processes that are responsible for the heating of stellar atmospheres, and use them to explain the observed range of stellar activity. The focus of this paper is on the second problem (Sect. 2) and, in particular, on wave heating mechanisms (Sect. 3), which have recently been used to construct theoretical models of stellar chromospheres (Sect. 4). Because of discrepancies between these models and observations, additional (non-wave) heating is required in the most upper regions of stellar chromospheres and in stellar transition regions (Sect. 5).

\section{Energy requirements and heating mechanisms}

The observed increase of temperature in stellar atmospheric layers located above the photosphere demands continuous energy supplies to maintain the required heating. Formally, the non-radiative energy can be supplied from the photosphere or below, or from the corona, or be generated in the chromosphere and transition region. Semiempirically determined energy requirements of different solar chromospheric and coronal features have been recently summarized by Ulmschneider \& Musielak (2003, see their Table 2) who concluded that the required non-radiative energy is of the order of several times $10^{7} \mathrm{erg} \mathrm{cm}^{-2} \mathrm{~s}^{-1}$ and is roughly a factor of ten lower than the amount of energy supplied by different heating mechanisms (see Sect. 3).

According to Narain \& Ulmschneider (1996), the various proposed heating mechanisms can be divided into two main categories: hydrodynamic (acoustic and pulsational waves) and magnetic (MHD waves and magnetic field dissipation). Presently, neither observations nor theory have been able to definitively determine which one of these two general classes of models dominates in the atmospheric heating (see, however, Sect. 4 and 5). Therefore, one may assume that the acoustic and magnetic wave heating mechanisms dominate in stellar chromospheres and that all wave energy needed for this heating is generated in the stellar convection zones. For the wave heating models, the key theoretical problems are: (1) to identify waves which are the most important for the heating; (2) to estimate how efficiently and where these waves are generated; and (3) to calculate how these waves propagate and dissipate energy and momentum.

A large number of theoretical papers have been published on these subjects (e.g., Narain \& Ulmschneider 1996; Ulmschneider \& Musielak 2003) and wave heating theory has been used to construct theoretical chromospheric models and compare them directly with the observational data (e.g., Buchholz et al. 1998; Cuntz, Ulmschneider \& Musielak 1998; Cuntz et al. 1999; Ulmschneider et al. 
2001a; and Fawzy et al. 2002a,b,c). These models require the wave energy flux carried by different waves to be specified at the atmospheric height where the calculations begin. Since the models are theoretical, the amount of energy carried by the waves must also be evaluated theoretically.

\section{Wave generation and energy available for heating}

The most prominent source of the non-radiative energy required to heat stellar atmospheres are obviously stellar convection zones. The fact that highly turbulent motions existing in these convective zones can be responsible for the generation of acoustic and magnetic tube waves has been known for many years (e.g., Musielak 1991; Ulmschneider \& Musielak 2003).

\subsection{The Lighthill-Stein theory of sound generation}

In its original version, Lighthill's theory of sound generation (Lighthill 1952) deals with the acoustic power output produced by a highly localized source of acoustic waves represented by a turbulent jet. The power is calculated at large distances from the source where the waves are freely propagating. The basic idea is to use the set of fundamental hydrodynamic equations and derive an inhomogeneous wave equation for a single wave variable, typically, wave density $\rho$ or pressure $p$. The wave equation is derived by collecting all linear and nonlinear terms on the left-hand side (the propagator) and the right-hand side (the source function), respectively. This gives $\hat{L}[\rho]=\hat{S}\left[u_{t}\right]$, where the acoustic wave propagator is $\hat{L}=\partial^{2} / \partial t^{2}-c_{S}^{2} \nabla^{2}, c_{S}$ is the sound speed, and $u_{t}$ represents the turbulent velocity. It is assumed that the source function $\hat{S}\left[u_{t}\right]$ can be determined by the properties of a turbulent flow. The main result is that the fluid motions generating linear acoustic waves behave as a volume distribution of acoustic quadrupoles, which means that $\hat{S}\left[u_{t}\right]=S_{q u}$. It must be noted that Lighthill's theory has been verified experimentally and its predictions were found to be in very good agreement with the experimental data (Goldstein 1976).

The Lighthill theory deals only with homogeneous media and treats turbulence as isotropic, homogeneous and decaying in time. All these assumptions are, however, of dubious validity for stellar convective zones where gradients in density, temperature and pressure occur, and the turbulence is driven by the fluctuating buoyancy force (e.g., Goldreich \& Kumar 1988). Stein (1967) followed the work done by Unno \& Kato (1962), and Moore \& Spiegel (1964) and modified the theory to be applicable to more realistic physical conditions occuring in stellar convective zones. He considered an isothermal and stratified atmosphere and included effects caused by the non-decaying turbulence, although the backreaction of generated waves on the turbulence was not accounted for. He derived and solved the following inhomogeneous wave equation

$$
\hat{L}_{A}\left[p_{1}\right]=\hat{S}_{A}\left[p_{o}, u_{t}\right]
$$

where $p_{1}=p / \sqrt{p_{o}}$ and

$$
\hat{L}_{A}=\frac{\partial^{2}}{\partial t^{2}}-c_{S}^{2} \nabla^{2}+\Omega_{S}^{2}-c_{S}^{2} \Omega_{B V}^{2}\left(\frac{\partial}{\partial t}\right)^{-2}\left(\frac{\partial^{2}}{\partial x^{2}}+\frac{\partial^{2}}{\partial y^{2}}\right)
$$


$p_{o}$ is the background pressure, $\Omega_{S}=c_{S} / 2 H$ (with $H$ being the pressure scale height) is the acoustic cutoff frequency, and $\Omega_{B V}=c_{S} \sqrt{\gamma-1} / \gamma H$ is the BruntVäisälä frequency; note that acoustic waves are propagating only for frequencies $\omega>\Omega_{S}$ with $\Omega_{S}>\Omega_{B V}$. The source function $\hat{S}_{A}\left[p_{o}, u_{t}\right]$ (see Stein 1967, Eq. 11 ) is calculated by making the multipole expansion which allows separating contributions from different wave sources and writing the source function in the form: $\hat{S}_{A}\left[p_{o}, u_{t}\right]=S_{q u d}+S_{d i p}+S_{m o n}$, where $S_{q u d} \sim \omega^{4}, S_{d i p} \sim \omega^{2} \Omega_{S}^{2}$ and $S_{m o n} \sim \Omega_{S}^{4}$. The dipole and monopole source terms describe the conversion of kinetic energy into acoustic energy resulting from forcing the mass and momentum in a fixed region of space to fluctuate, respectively, and are produced by the external gravitational force.

The Lighthill-Stein theory was used to calculate solar and stellar acoustic wave energy fluxes (e.g., Renzini et al. 1977; Bohn (1984). However, as shown by Musielak et al. (1994) these results are incorrect, mainly because several fundamental assumptions of the theory turned out to be physically unjustified. The theory has been corrected by incorporating an improved description of the spatial and temporal spectrum of the turbulent convection (see Musielak et al. 1994) and used to compute stellar acoustic wave energy spectra and fluxes (e.g., Ulmschneider, Theurer \& Musielak 1996; Ulmschneider et al. 1999). The fluxes computed for late-type stars with $\log g=4$ are shown in Fig. 3.

\subsection{Generation of magnetic tube waves}

The highly intermittent spatial magnetic (flux tube) structures (see Fig. 1) have also been incorporated into the theory of wave generation. An isolated magnetic flux tube, which is assumed to be thin and with a circular cross-section, is considered to be embedded in a magnetic field-free, turbulent, compressible and isothermal external medium. The tube is in temperature equilibrium with the external medium and it remains vertically oriented under the assumption that there are strong buoyancy forces in the upper layers of the convection zones. Because of this vertical orientation, the three fundamental tube wave modes (see Fig. 1) can be separated and, as a result, the generation of longitudinal (Musielak, Rosner \& Ulmschneider 1989; Musielak et al. 1995), transverse (Musielak \& Ulmschneider 2001) and torsional (Noble, Musielak \& Ulmschneider 2003a) tube waves can be treated independently.

To describe the interaction between the tube and the external turbulent motions, an inhomogeneous wave equation of the form given by Eq. (1) is derived from the set of ideal MHD equations for each tube wave. The source functions, which are introduced into the theory through the horizontal pressure balance for the tube, have different forms that depend on both the external motions and the form of boundary conditions at the tube surface. In the following, the explicit form of the propagator and source function is given for each tube wave. The choice of the wave variable is also specified, however, it has no effect on the results because in the considered approach the behavior of all wave variables is identical.

For longitudinal tube waves, the inhomogeneous wave equation is derived for the wave pressure perturbation $p_{2}=p / \sqrt{\rho_{o} B_{o}}$, where $\rho_{o}$ is the gas density inside the tube and $B_{o}$ is the tube magnetic field. The normalization factor $\sqrt{\rho_{o} B_{o}}$ is chosen so that the cutoff frequency $\Omega_{D}$ for longitudinal tube waves 

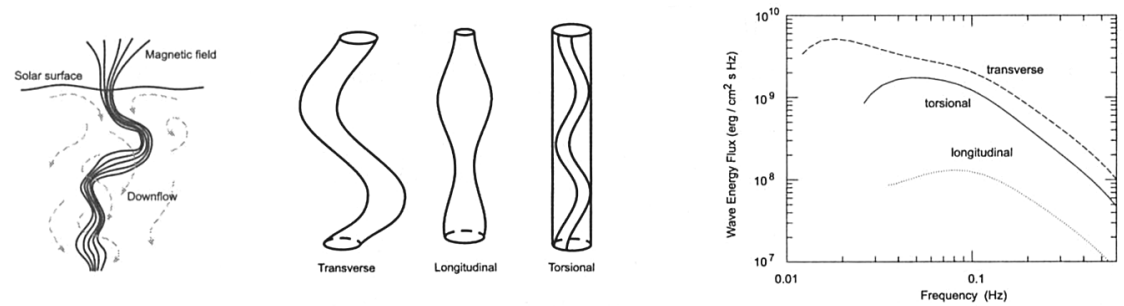

Figure 1. Generation of magnetic tube waves by solar convective motions (left), after Parker (1981), three fundamental tube wave modes (middle), and the wave energy spectra carried by longitudinal, transverse and torsional tube waves in the solar atmosphere (right), after Noble, Musielak \& Ulmschneider (2003a)

(Defouw 1976) is explicitly displayed in the propagator, which is given by

$$
\hat{L}_{T}\left[p_{2}\right]=\left[\frac{\partial^{2}}{\partial t^{2}}-c_{T}^{2} \frac{\partial^{2}}{\partial z^{2}}+\Omega_{D}^{2}\right] p_{2},
$$

where the tube speed is $c_{T}=c_{S} c_{A} / \sqrt{c_{S}^{2}+c_{A}^{2}}$, with $c_{A}=B_{o} / \sqrt{4 \pi \rho_{o}}$ being the Alfvén velocity, $\Omega_{D}=c_{T} \sqrt{9 / 16-1 / 2 \gamma+c_{S}^{2}(\gamma-1) / \gamma^{2} c_{A}^{2}} / H$. According to Musielak et al. (1995), the source function is given by

$$
\hat{S}_{T}\left[u_{t}^{2}\right]=\frac{\rho_{e}}{2 \sqrt{\rho_{o} B_{o}}} \frac{c_{T}^{2}}{c_{A}^{2}}\left(\frac{\partial^{2}}{\partial t^{2}}+\Omega_{B V}^{2}\right) u_{t}^{2},
$$

where $u_{t}^{2}(z, t)$ represents the external turbulent flow field.

The inhomogeneous wave equation for transverse (kink) tube waves is derived for the wave velocity perturbation $v_{1}=v_{x} \rho_{o}^{1 / 4}$ and the propagator is given by

$$
\hat{L}_{K}\left[v_{1}\right]=\left[\frac{\partial^{2}}{\partial t^{2}}-c_{K}^{2} \frac{\partial^{2}}{\partial z^{2}}+\Omega_{K}^{2}\right] v_{1},
$$

where $c_{K}=B_{o} / \sqrt{4 \pi\left(\rho_{e}+\rho_{o}\right)}$, with $\rho_{e}$ being the gas density outside the tube, and $\Omega_{K}=c_{K} / 4 H$ being the cutoff frequency for these waves (Spruit 1981). As shown by Musielak \& Ulmschneider (2001), the source function can be written in the following form:

$$
\begin{gathered}
\hat{S}_{K}\left[u_{x}, u_{z}\right]=\frac{\rho_{o}^{1 / 4} \rho_{e}}{\rho_{o}+\rho_{e}}\left[\frac{\partial u_{x}}{\partial t}+\frac{\partial^{2} u_{x}}{\partial t^{2}}\left(\frac{\partial}{\partial t}\right)^{-1}+g \frac{\partial u_{x}}{\partial z}\left(\frac{\partial}{\partial t}\right)^{-1}+g\left(\frac{\partial}{\partial t}\right)^{-1} \frac{\partial u_{x}}{\partial z}\right] \\
\times\left(\frac{1}{H}-\frac{\partial}{\partial z}\right) u_{z}
\end{gathered}
$$

where $u_{x}$ and $u_{z}$ are the $\mathrm{x}$ and z-components of the external turbulent velocity. 
Finally, for torsional tube waves the inhomogeneous wave equation is derived for the wave velocity perturbation $v_{2}=v_{\phi} \rho_{o}^{1 / 4}$ and the propagator is given by

$$
\hat{L}_{A}\left[v_{2}\right]=\left[\frac{\partial^{2}}{\partial t^{2}}-c_{A}^{2} \frac{\partial^{2}}{\partial z^{2}}+\Omega_{A}^{2}\right] v_{2},
$$

where $\Omega_{A}=c_{A} / 4 H$ is the cutoff frequency for these waves (Noble et al. 2003a). The source function can be written in the following form:

$$
\hat{S}_{A}\left[u_{\phi}, u_{z}\right]=\rho_{o}^{1 / 4} \frac{\rho_{e}}{\rho_{o}}\left[\frac{\partial u_{\phi}}{\partial t}+\frac{\partial^{2} u_{\phi}}{\partial t^{2}}\left(\frac{\partial}{\partial t}\right)^{-1}\right]\left(\frac{1}{H}-\frac{\partial}{\partial z}\right) u_{z}
$$

where $u_{\phi}$ and $u_{z}$ are the $\phi$ and z-components of the external turbulent velocity.

The derived inhomogeneous wave equations can easily be solved by using a space and time Fourier transform because all coefficients in these equations are constant. The mean generation rate of each tube wave is calculated by expressing the wave energy flux in terms of Fourier transforms of the source function, averaging over time and space, and evaluating analytically the asymptotic Fourier transforms. Since the source function is expressed in terms of the second-order turbulent velocity correlations, the Fourier transform of the latter becomes a convolution intergral, which is also evaluated analytically for a given spatial (modified Kolmogorov) and temporal (modified Gaussian) turbulent energy spectrum (Musielak et al. 1994). The resulting source function describes dipole sources of the generation of magnetic tube waves.

Examples of the longitudinal, transverse and torsional wave energy spectra generated in the solar convection zone are given in Fig. 1, which shows that the generation rates of transverse and torsional tube waves are much higher than that for longitudinal waves. Similar calculations have been performed for latetype stars by Musielak et al. (2000, 2002), Musielak \& Ulmschneider (2002a,b), and Noble et al. (2003b). The wave energy fluxes obtained by these authors for late-type stars with different $T_{\text {eff }}$ but the same $\log \mathrm{g}=4$ are presented in Fig. 2. Note that the presented spectra and fluxes are computed for one magnetic flux tube and that they do not depend on stellar filling factors.

The analytical approach, which is limited to linear waves but takes into account the correlation effects, has been supplemented by a numerical treatment that allows considering nonlinear longitudinal waves, however, does not account for the correlation effects (Ulmschneider \& Musielak 1998). Because of the difference in these two approaches, the analytically and numerically calculated fluxes can be seen as lower and upper bounds for the realistic stellar wave energy fluxes. The numerical approach has been used by Ulmschneider et al. (2001b) to calculate the amount of energy carried by longitudinal tube waves in atmospheres of selected late-type dwarfs and subgiants. Since the calculations have been performed only for a narrow range of $T_{\text {eff }}$, the numerical wave energy fluxes are not included in Fig. 2.

There have also been several attempts to estimate the amount of energy carried by nonlinear transverse tube waves in the solar atmosphere. Various authors have based their estimates on observational data (Muller et al. 1994), others have used analytical (Choudhuri et al. 1993a, b) or numerical (Huang, Musielak \& Ulmschneider 1995) methods. The results were obtained for the Sun 


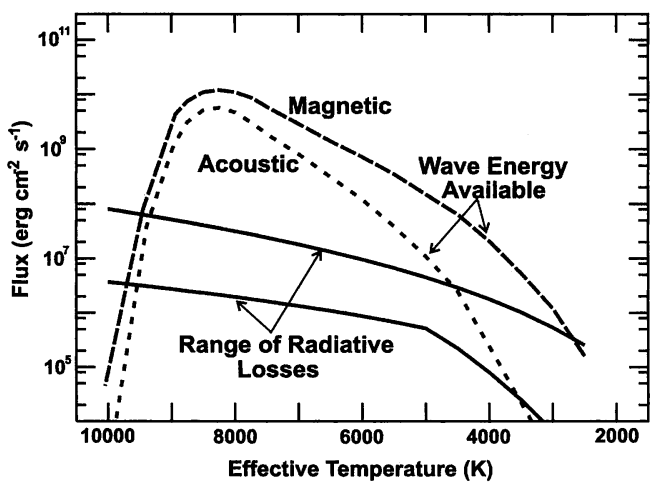

Figure 2. Stellar acoustic and magnetic (longitudinal + transverse + torsional) wave energy fluxes calculated analytically for stars with $\log \mathrm{g}=4$ (after Ulmschneider et al. 1996 and Noble et al. 2003a) are compared to the observationally established range of radiative losses for these stars (after Fazwy et al. 2002c).

and they show that transverse tube wave are generated 30 or even 40 times more efficiently than longitudinal tube waves, these fluxes are not included in Fig. 2.

\subsection{Wave energy available versus radiative losses}

Comparison between stellar acoustic and magnetic wave energy fluxes and the observed radiative losses is shown in Fig. 2. The analytical methods described above were used to compute the acoustic and magnetic (longitudinal + transverse + torsional) for stars with $\log g=4$ and different $T_{\text {eff }}$ (see Noble et al. 2003a). The radiative losses were taken from Fawzy et al. (2002c). The comparison clearly shows that there is a significant excess of the wave energy available for the acoustic heating (the acoustic fluxes should be compared only to the lower bound of the radiative losses) as well as for the magnetic heating (the magnetic fluxes should be higher than, or comparable to, the upper bound of the radiative losses) for stars with $\mathrm{T}_{\text {eff }}$ ranging from $4000 \mathrm{~K}$ to $9000 \mathrm{~K}$. Now, it remains to be determined whether these fluxes are sufficient to heat stellar chromospheres and transition regions.

\section{Wave-based models of stellar chromospheres}

Using the calculated wave energy fluxes, new theoretical, time-dependent and two-component stellar chromospheric models have been computed by Ulmschneider et al. (2001a) and Fawzy et al. (2002a,b,c). These models are based exclusively on wave heating and they consist of non-magnetic regions heated by acoustic waves and magnetic flux tube regions heated by magnetic (longitudinal and transverse) tube waves. They require specifying only four basic stellar parameters, namely, effective temperature $T_{\text {eff }}$, gravity $g$, metallicity $Z_{\mathrm{M}}$ and rotation period $P_{\text {rot }}$. Because the first-principle theory of stellar dynamos is not yet available (e.g., Weiss 1994), the rotation period is replaced by the magnetic 


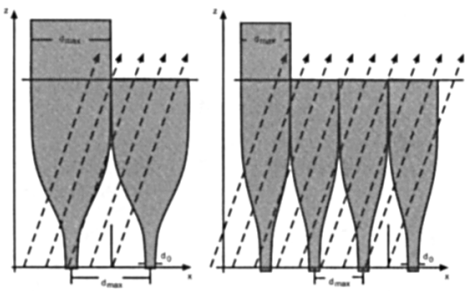

Figure 3. Models of uniformly distributed magnetic flux tubes over the stellar surface for two different values of the filling factor.

filling factor $f$, which is treated as the only free parameter in these models. By specifying the magnetic filling factor (see Fig. 3), theoretical models of stellar atmospheres with different chromospheric activity have been calculated.

A time-dependent MHD code based on the method of characteristics is used to compute the propagation of acoustic waves in a plane-parallel atmosphere (e.g., Buchholz et al. 1998) and longitudinal waves along magnetic flux tubes (e.g., Herbold et al. 1985). Simultaneously with these wave calculations, radiative losses in the $\mathrm{H}^{-}$continuum and in the $\mathrm{Mg}$ II and $\mathrm{Ca}$ II emission lines by solving the appropriate radiative transfer equations together with the statistical equilibrium equations for NLTE populations. The time-dependent energy balance between the wave dissipation due to shocks and the emitted radiative losses determines the local values of temperature, density and pressure in the magnetic and non-magnetic stellar atmosphere regions. These models have been used to predict the theoretical level of stellar chromospheric activity and compare it to the observational data (see Fig. 4).

The comparison between theoretically predicted stellar chromospheric activity and that observed clearly shows that the wave heating alone cannot fully account for the observed range of activity (see Fig. 4). However, the results imply that the base of stellar chromospheres is heated by acoustic waves, and that the heating of the middle and upper chromospheric layers is dominated by magnetic waves associated with magnetic flux tubes and other non-wave (e.g., reconnective) heating mechanisms; the latter are required to explain the structure of the highest layers of stellar chromospheres and stellar transition regions.

The described chromospheric models have several limitations, which could be responsible for the discrepancies between the models and observations. First, the wave propagation and dissipation is essentially one-dimensional in the vertical direction. It has been recently suggested by Kalkofen $(2003 a, b)$ that both acoustic and magnetic waves should be treated as spherical waves produced by nonuniformly distributed sources; this would lead to more wave energy being available for the heating in the upper layers. Second, only shocks formed by longitudinal tube waves are responsible for the heating of magnetic regions; a fraction of the energy carried by transverse tube waves becomes also available for this heating as a result of nonlinear mode-coupling of these waves to longitudinal waves (Ulmschneider et al. 1991). Recently, two other mechanisms of converting magnetic waves into heat have been studied. One of them is the resistive disspative heating proposed by Goodman (2000), and the other is damping of transverse waves due to collisions between ions and neutrals (De Pontieu, 

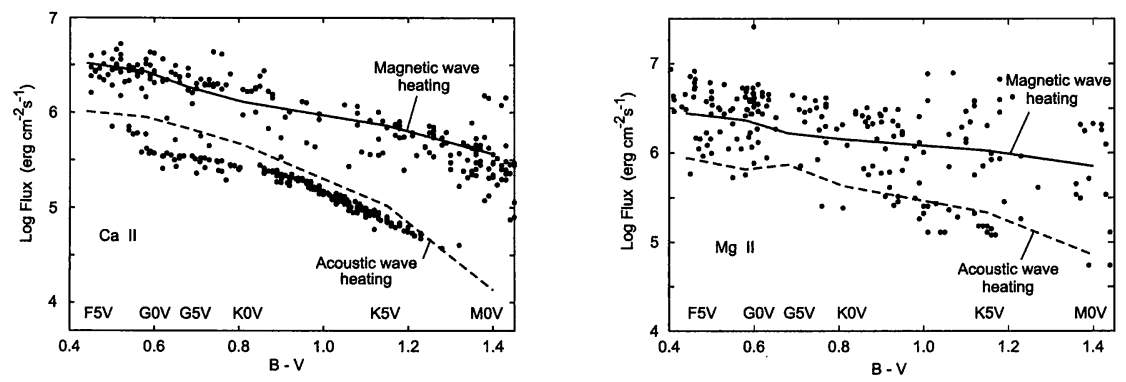

Figure 4. Total observed $\mathrm{Ca}$ II $\mathrm{H}+\mathrm{K}$ (left) and $\mathrm{Mg}$ II $\mathrm{h}+\mathrm{k}$ (right) line core emission fluxes (with subtracted photospheric background) for late-type stars of different activity are shown as dots and compared with theoretical emission fluxes computed from models based on pure acoustic wave heating (dashed line) and on acoustic / magnetic wave heating (solid line) with the filling factor $f=0.4$ at the stellar surface - after Fawzy et al. (2002b).

Martens \& Hudson 2001). Magnetic waves can also be dissipated by resonance absorption and phase mixing (e.g., Davila 1991; Roberst 1991; Priest \& Forbes 2000), and by turbulent and viscous heating (e.g., Strauss 1991) in special magnetic field geometries. Including these mechanisms in the theoretical models (as challenging as it might be) would lead to more efficient wave heating. Third, the energy carried by torsional tube waves (see Fig. 1) must also be taken into account in the models.

Finally, it is important to emphasize that time-dependent hydrogen ionization is not included in these chromospheric models, which means that they become inaccurate at chromospheric heights where hydrogen is ionized. Theoretical models with hydrogen ionization describing the solar atmosphere have been constructed by Carlsson \& Stein (1997). Recently, Rammacher \& Ulmschneider (2003) have incorporated time-dependent hydrogen ionization into the above chromospheric models. Application of these newly improved models to $\tau$ Ceti, a G8 V star with extremely low chromospheric activity, shows that the models can now account much more precisely for the levels of the basal flux than those shown in Fig. 4 (Rammacher \& Cuntz 2003).

\section{Other Heating Mechanisms}

The presented results clearly show that the wave heating mechanism must be supplemented (or even replaced) by other magnetic (the so-called DC-heating) mechanisms. As two examples of these mechanisms, one may consider two different situation where current sheets exist. The first one is an arcade system (Priest 1991), which by slow motions is laterally compressed and develops a current sheet, where the oppositely directed magnetic field lines reconnect. The energy is released at these reconnection sides and the local medium is heated to high temperatures. The other example, after Parker (1992), is a tangled and braided web of atmospheric magnetic field lines created by the continual 
shuffling and intermixing of their foot points by slow photospheric motions. At many locations in the web oppositely directed fields occur, giving rise to local current sheets, which by reconnection release the magnetic field energy. These small-scale reconnection events are explosive and often referred to as microflares or nanoflares (Parker 1988; Mendoza-Briceno, Erdélyi, \& Sigalotti 2002).

According to SOHO observations, small magnetic loops are being perpetually generated in the solar atmosphere and then very quickly disappear by releasing their energy through magnetic reconnection. These short-lived loops (microflares) form what is called a "magnetic carpet" and its energy content seems to be sufficient to heat the solar corona (Schrijver et al. 1997). Priest, Heyvaerts and Title (2002) have studied coronal heating driven by this magnetic carpet and introduced a flux-tube tectonics model to explain the formation and dissipation of magnetic current sheets.

The problem of heating of stellar atmospheres by small-scale explosive events (microflares) has been studied by Wood, Linsky, \& Ayres (1997) who observed C IV and Si IV transition layer lines in some active late-type stars. They have found that the total line profiles can be explained as a combination of a very broad profile attributed to microflares and a narrow profile attributed to waves, which means that both wave and non-wave heating mechanisms operate in the upper layers of stellar atmospheres; the theoretical results described in this paper are consistent with this conclusion.

\section{Conclusions}

The presented results show that the base of stellar chromospheres is heated by acoustic waves whereas the heating of the middle and upper chromospheric layers is dominated by magnetic waves associated with magnetic flux tubes.

To explain the structure of the most upper layers of stellar chromospheres and stellar transition regions other non-wave heating mechanisms are required. The most promising are small-scale explosive events known as micro(nano)flares.

From a theoretical point of view, the next most challenging step will be to incorporate these small-scale reconnection events into the existing wave-based theoretical models.

Acknowledgements This work was supported by NSF under grant ATM-0087184 and by the Alexander von Humboldt Foundation.

\section{References}

Baliunas, S.L., et al. 1995, ApJ, 438, 269

Bohn, H.U. 1984, A\&A, 136, 338.

Buchholz, B., Ulmschneider, P. \& Cuntz, M. 1998, ApJ, 494, 700

Carlsson, M., \& Stein, R.F. 1997, ApJ, 481, 500

Choudhuri, A.R., Auffret, H. \& Priest, E.R. 1993a, Sol. Phys., 143, 49

Choudhuri, A.R., Dikpati, M. \& Banerjee, D. 1993b, ApJ, 413, 811

Cuntz, M., Ulmschneider, P., \& Musielak, Z.E. 1998, ApJ, 493, L117 
Cuntz, M., Rammacher, W., Ulmschneider, P., Musielak, Z.E. \& Saar, S.H. 1999, ApJ, 522, 1053

Davila, J.M. 1991, in Mechanisms of Chromospheric and Coronal Heating, eds. P. Ulmschneider, E.R. Priest, R. Rosner, Springer, Heidelberg, 464

Defouw, R.J. 1976, ApJ, 209, 266

De Pontieu, B., Martens, P.C.H., \& Hudson, H.S. 2001, ApJ, 558, 859

Donati, J.F. 1999, MNRAS 302, 457

Dupree, A.K., Whitney, B.A., \& Pasquini, L. 1999, ApJ, 520, 751

Fawzy, D., Rammacher, W., Ulmschneider, P., Musielak, Z.E. \& Stȩpień, K. 2002a, A\&A, 386, 971

Fawzy, D., Ulmschneider, P., Stẹpień, K., Musielak, Z.E. \& Rammacher, W. 2002b, A\&A, 386, 983

Fawzy, D., Stępień, K., Ulmschneider, P., Rammacher, W., \& Musielak, Z.E. 2002c, A\&A, 386, 994

Goldreich, P., \& Kumar, P. 1988, ApJ, 326, 462

Goldstein, M.A. 1976, Aeroacoustic, McGraw-Hill, New York, 94

Goodman, M.L. 2000, ApJ, 533, 501

Herbold, G., Ulmschneider, P., Spruit, H.C. \& Rosner, R. 1995, A\&A, 145, 157

Huang, P., Musielak, Z.E. \& Ulmschneider, P. 1995, A\&A, 297, 579

Jordan, C. 1997, Astr. Geophys., 38, 10

Kalkofen, W. 2003a, in Current Theoretical Models and Future High Resolution Solar Observations, eds. A.A. Pevtsov, H. Uitenbroek, APS Conf. Ser., 286,385

Kalkofen, W. 2003b, Astron. Nachr., 324, 409

Lighthill, M.J. 1952, Proc. R. Soc. Lond. A, 211, 564

Linsky, J.L. 1991, in Mechanisms of Chromospheric and Coronal Heating, eds. P. Ulmschneider, E.R. Priest, R. Rosner, Springer, Heidelberg, 166

Linsky, J.L. et al. 1992, in Cool Stars, Stellar Systems, and the Sun, eds. M.S. Giampapa, J.A. Bookbinder, ASP Conf. Ser. 26, 106

Mendoza-Briceno, C.A., Erdélyi, R., \& Sigalotti, L.G. 2002, ApJ, 579, L49

Moore, D. W., \& Spiegel, E. A., 1964, ApJ, 139, 48

Muller R., Roudier Th., Vigneau J., \& Auffret H., 1994, A\&A 283, 232

Musielak, Z.E. 1991, in Mechanisms of Chromospheric and Coronal Heating, eds. P. Ulmschneider, E.R. Priest, R. Rosner, R., Springer, Heidelberg, 369

Musielak, Z.E., Rosner, R., Gail, H.P. \& Ulmschneider, P. 1995, ApJ, 448, 865

Musielak, Z.E., Rosner, R., Stein, R.F. \& Ulmschneider, P. 1994, ApJ, 423, 474

Musielak, Z.E., Rosner, R. \& Ulmschneider, P. 1989, ApJ, 337, 470

Musielak, Z.E., Rosner, R. \& Ulmschneider, P. 2000, ApJ, 541, 410

Musielak, Z.E., Rosner, R. \& Ulmschneider, P. 2002, ApJ, 573, 418

Musielak, Z.E. \& Ulmschneider, P. 2001, A\&A, 370, 541

Musielak, Z.E. \& Ulmschneider, P. 2002a, A\&A, 386, 606

Musielak, Z.E. \& Ulmschneider, P. 2002b, A\&A, 386, 615 
Narain, U. \& Ulmschneider, P. 1996, Space Sci. Rev., 75, 453

Noble, M.W., Musielak, Z.E., \& Ulmschneider, P. 2003, A\&A, 409, 1085

Noble, M.W., Musielak, Z.E., \& Ulmschneider, P. 2003a, A\&A, submitted

Parker, E.N. 1981, in Solar Phenomena in Stars and Stellar Systems, Proc. Adv.

Study Inst. Bonas, eds. Bonnet, R.M., Dupree, A.K., D. Reidel Pub., 33

Parker, E.N. 1988, ApJ, 330, 474

Parker, E.N. 1992, J. Geophys. Res., 97, 4311

Priest, E.R. 1991, in Mechanisms of Chromospheric and Coronal Heating, eds.

P. Ulmschneider, E.R. Priest, R. Rosner, Springer, Heidelberg, 520

Priest, E.R., \& Forbes, T.G. 2000, J. Geophys. Res., 91, 5579

Priest, E.R., Heyvaerts, J.F., \& Title, A.M. 2002, ApJ, 576, 533

Rammacher, W., \& Cuntz, M. 2003, ApJ, 594, L51

Rammacher, W., \& Ulmschneider, P. 2003, ApJ, 589, 988

Renzini, A., Cacciari, C., Ulmschneider, P., \& Schmitz, F. 1977, A\&A, 61, 39

Rutten, R.G.M., Schrijver, C.J., Lemmens, A.F.P. \& Zwaan, C. 1991, A\&A, 252,203

Saar, S.H. 1996, in Stellar Surface Structure, eds. K. Strassmeier, J.L. Linsky, Kluwer, Dordrecht, 237

Saar, S.H. 2001, in Cool Stars, Stellar Systems, and the Sun, eds. R.J.G. Lopez, R. Rebolo, M.R.Z. Osorio, ASP Conf. Ser., 223, 292

Schrijver, C.J. 1987, A\&A, 172, 111

Schrijver, C.J., Title, A.M., van Ballegooijen, A.A., Hagenaaar, H.J. \& Shine, R.A. 1997, ApJ, 487, 424

Solanki, S.K. 1992, Space Sci. Rev., 55, 92

Spruit H.C. 1981, A\&A, 98, 155

Stein, R.F. 1967, Sol. Phys., 2, 385

Stȩpień, K. 1994, A\&A, 292, 191

Strauss, H.R. 1991, Geophys. Res. Let., 18, 77

Ulmschneider, P., Fawzy D., Musielak Z.E. \& Stępień K. 2001a, ApJ, 559, L167

Ulmschneider, P. \& Musielak, Z.E. 1998, A\&A, 338, 311

Ulmschneider, P., \& Musielak, Z.E. 2003, in Current Theoretical Models and

Future High Resolution Solar Observations, eds. A.A. Pevtsov, H. Uitenbroek, APS Conf. Ser., 286, 363

Ulmschneider, P., Musielak, Z.E. \& Fawzy, D.E. 2001b, A\&A, 374, 662

Ulmschneider, P., Theurer, J. \& Musielak, Z.E. 1996, A\&A, 315, 212

Ulmschneider, P., Theurer, J., Musielak, Z.E. \& Kurucz, R. 1999, A\&A, 347, 243

Ulmschneider, P., Zähringer, K. \& Musielak, Z.E. 1991, A\&A, 241, 625

Unno, W., \& Kato, S. 1962, Publ. Astr. Soc. Japan, 14, 417

Weiss, N.O. 1994, in Lectures on Solar and Planetary Dynamos, eds. M.R.E.

Proctor, A.D. Gilbert, Cambridge Uni. Press, 59

Wood, B.E., Linsky, J.L., \& Ayres, T.R. 1997, ApJ, 478, 745 